\title{
Viscosity iteration methods for a split feasibility problem and a mixed equilibrium problem in a Hilbert space
}

\author{
Bin-Chao Deng ${ }^{1 *}$, Tong Chen ${ }^{1}$ and Qiao-Li Dong ${ }^{2}$
}

\section{"Correspondence:}

dbchao1985@yahoo.com.cn

'School of Management, Tianjin University, Tianjin, 300072, China Full list of author information is available at the end of the article

\begin{abstract}
In this paper, we consider and analyze two viscosity iteration algorithms (one implicit and one explicit) for finding a common element of the solution set $\operatorname{MEP}\left(F_{1}, F_{2}\right)$ of a mixed equilibrium problem and the set $\Gamma$ of a split feasibility problem in a real Hilbert space. Furthermore, we derive the strong convergence of a viscosity iteration algorithm to an element of $\operatorname{MEP}\left(F_{1}, F_{2}\right) \cap \Gamma$ under mild assumptions.
\end{abstract}

Keywords: mixed equilibrium problem; split feasibility problem; firmly nonexpansive; inverse strongly monotone; projection

\section{Introduction}

The split feasibility problem (SFP) in finite-dimensional Hilbert spaces was first introduced by Censor and Elfving [1] for modeling inverse problems which arise from phase retrievals and in medical image reconstruction [2]. In this paper we work in the framework of infinite-dimensional Hilbert spaces. In this setting, the split feasibility (SFP) is formulated as finding a point $x^{*}$ with the property

$$
x^{\prime \prime} \in C \text { and } A x^{\prime \prime} \in Q \text {, }
$$

where $C$ and $D$ are the nonempty closed convex subsets of the infinite-dimensional real Hilbert spaces $H_{1}$ and $H_{2}$, and $A: H_{1} \rightarrow H_{2}$ is a bounded linear operator. For related works, please refer to [2-8].

Let $H$ be a real Hilbert space whose inner product and norm are denoted by $\langle\cdot, \cdot\rangle$ and $\|\cdot\|$, respectively. Let $C$ be a nonempty closed convex subset of $H$, and let $F$ be a bifunction of $C \times C$ into $\mathbb{R}$ which is the set of real numbers. The equilibrium problem for $F: C \times C \rightarrow \mathbb{R}$ is to find $x \in C$ such that

$$
F(x, y) \geq 0, \quad \forall y \in C
$$

The set of solutions of (1.1) is denoted by $\mathrm{EP}(F)$. Equilibrium problems theory has emerged as an interesting and fascinating branch of applicable mathematics. The mixed equilibrium problem is as follows:

$$
\text { Find } x \in C: F_{1}(x, y)+F_{2}(x, y)+\langle A x, x-y\rangle \geq 0, \quad \forall y \in C .
$$


In the sequel, we indicate by $\operatorname{MEP}\left(F_{1}, F_{2}, A\right)$ the set of solutions of our mixed equilibrium problem. If $A=0$, we denote $\operatorname{MEP}\left(F_{1}, F_{2}, 0\right)$ by $\operatorname{MEP}\left(F_{1}, F_{2}\right)$. The mixed equilibrium problem (1.3) has become a rich source of inspiration and motivation for the study of a large number of problems arising in economics, optimization problems, variational inequalities, minimax problem, Nash equilibrium problem in noncooperative games and others (e.g., [9-14]).

It is our purpose in this paper to consider and analyze two viscosity iteration algorithms (one implicit and one explicit) for finding a common element of a solution set $\Gamma$ of the split feasibility problem (1.1) and a set $\operatorname{MEP}\left(F_{1}, F_{2}\right)$ of the mixed equilibrium problem (1.3) in a real Hilbert space. Furthermore, we prove that the proposed viscosity iteration methods converge strongly to a particular solution of the mixed equilibrium problem (1.3) and the split feasibility problem (1.1).

\section{Preliminaries}

Assume $H$ is a Hilbert space and $C$ is a nonempty closed convex subset of $H$. The projection, denoted by $P_{C}$, from $H$ onto $C$ assigns for each $x \in H$ the unique point $P_{C} x \in C$ so that

$$
\left\|x-P_{C} x\right\|=\inf \{\|x-y\|: y \in C\} .
$$

Proposition 2.1 (Basic properties of projections [15])

(i) $\left\langle x-P_{C} x, y-P_{C} x\right\rangle \leq 0$ for all $x \in H$ and $y \in C$;

(ii) $\left\langle x-y, P_{C} x-P_{C} y\right\rangle \geq\left\|P_{C} x-P_{C} x\right\|^{2}$ for all $x, y \in H$;

(iii) $\left\|x-P_{C} x\right\|^{2} \leq\|x-y\|^{2}-\left\|y-P_{C} x\right\|^{2}$ for all $x \in H$ and $y \in C$.

We also consider some nonlinear operators which are introduced in the following.

Definition 2.2 Let $A: C \rightarrow H$ be a nonlinear mapping. $A$ is said to be

(i) Monotone if

$$
\langle A x-A y, x-y\rangle \geq 0, \quad \forall x, y \in C .
$$

(ii) Strongly monotone if there exists a constant $\alpha>0$ such that

$$
\langle A x-A y, x-y\rangle \geq \alpha\|x-y\|^{2}, \quad \forall x, y \in C .
$$

For such a case, $A$ is said to be $\alpha$-strongly-monotone.

(iii) Inverse-strongly monotone (ism) if there exists a constant $\alpha>0$ such that

$$
\langle A x-A y, x-y\rangle \geq \alpha\|A x-A y\|^{2}, \quad \forall x, y \in C .
$$

For such a case, $A$ is said to be $\alpha$-inverse-strongly-monotone ( $\alpha$-ism).

(iv) $k$-Lipschitz continuous if there exists a constant $k \geq 0$ such that

$$
\|A x-A y\| \leq k\|x-y\|, \quad \forall x, y \in C .
$$


Remark 2.3 Let $F=I-\gamma f$, where $f$ is a $L$-Lipschitz mapping on $H$ with the coefficient $L>0, \gamma=\frac{1}{L}$. It is a simple matter to see that the operator $F$ is $(1-\gamma L)$-strongly monotone over $H$, i.e.,

$$
\langle F x-F y, x-y\rangle \geq(1-\gamma L)\|x-y\|^{2}, \quad \forall(x, y) \in H \times H .
$$

Definition 2.4 A mapping $T: H \rightarrow H$ is said to be

(a) nonexpansive if $\|T x-T y\| \leq\|x-y\|, \forall x, y \in H$;

(b) firmly nonexpansive if $2 T-I$ is nonexpansive. $T=(I+S) / 2$, where $S: H \rightarrow H$ is nonexpansive, Alternatively, $T$ is firmly nonexpansive if and only if

$$
\|T x-T y\|^{2} \leq\langle T x-T y, x-y\rangle, \quad x, y \in H
$$

(c) average if $T=(1-\epsilon) I+\epsilon S$, where $\epsilon \in(0,1)$ and $S: H \rightarrow H$ is nonexpansive. In this case, we also claimed that $T$ is $\epsilon$-averaged. A firmly nonexpansive mapping is $\frac{1}{2}$-averaged.

Proposition 2.5 ([3]) Let $T: H \rightarrow H$ be a given mapping.

(i) $T$ is nonexpansive if and only if the complement $I-T$ is $\frac{1}{2}-$ ism.

(ii) If $T$ is $v$-ism, then for $\gamma>0, \gamma T$ is $\frac{v}{\gamma}$-ism.

(iii) $T$ is averaged if and only if the complement $I-T$ is $v$-ism for $v>1 / 2$. Indeed, for $\alpha \in(0,1), T$ is $\alpha$-averaged if and only if $I-T$ is $\frac{1}{2 \alpha}$-ism.

Proposition 2.6 ([3]) Given operators $S, T, V: H \rightarrow H$.

(i) If $T=(1-\alpha) S+\alpha V$ for some $\alpha \in(0,1)$ and if $S$ is averaged and $V$ is nonexpansive, then $S$ is averaged.

(ii) $T$ is firmly nonexpansive if and only if the complement $I-T$ is firmly nonexpansive.

(iii) If $T=(1-\alpha) S+\alpha V$ for some $\alpha \in(0,1)$, $S$ is firmly nonexpansive and $V$ is nonexpansive, then $T$ is averaged.

(iv) The composite of finitely many averaged mappings is averaged. That is, if each of the mappings $\left\{T_{i}\right\}_{i=1}^{N}$ is averaged, then so is the composite $T_{1}, \ldots, T_{N}$. In particular, if $T_{1}$ is $\alpha_{1}$-averaged and $T_{2}$ is $\alpha_{2}$-averaged, where $\alpha_{1}, \alpha_{2} \in(0,1)$, then the composite $T_{1} T_{2}$ is $\alpha$-averaged, where $\alpha=\alpha_{1}+\alpha_{2}-\alpha_{1} \alpha_{2}$.

(v) If the mappings $\left\{T_{i}\right\}_{i=1}^{N}$ are averaged and have a common fixed point, then

$$
\bigcap_{i=1}^{N} F_{i x}\left(T_{i}\right)=F_{i x}\left(T_{1} \cdots T_{N}\right) .
$$

(Here the notation $F_{i x}(T)$ denotes the set of fixed points of the mapping $T$, that is, $F_{i x}(T)=\{x \in H: T x=x\}$.

Definition 2.7 A bifunction $g: C \times C \rightarrow \mathbb{R}$ is monotone if $g(x, y)+g(y, x) \leq 0, \forall x, y \in C$. A function $G: C \rightarrow \mathbb{R}$ is upper hemicontinuous if

$$
\limsup _{t \rightarrow \infty} G(t x+(1-t) y) \leq G(y)
$$


For solving the mixed equilibrium problem for a bifunction $F: C \times C \rightarrow \mathbb{R}$, let us assume that $F$ satisfies the following conditions:

(A1) $F(x, x)=0$ for all $x \in C$;

(A2) $F$ is monotone, that is, $F(x, y)+F(y, x) \leq 0$ for all $x, y \in C$;

(A3) for each $x, y, z \in C$,

$$
\lim _{t \rightarrow 0} F(t z+(1-t) x, y) \leq F(x, y)
$$

(A4) for each $x \in C, y \mapsto F(x, y)$ is convex and lower semicontinuous.

Lemma 2.8 ([16]) Let $C$ be a convex closed subset of a Hilbert space H. Let $F_{1}: C \times C \rightarrow \mathbb{R}$ be a bifunction such that

(f1) $F_{1}(x, x)=0, \forall x \in C$;

(f2) $F_{1}(x, \cdot)$ is monotone and supper hemicontinuous;

(f3) $F_{1}(\cdot, x)$ is lower semicontinuous and convex.

Let $F_{2}: C \times C \rightarrow \mathbb{R}$ be a bifunction such that

(h1) $F_{2}(x, x)=0, \forall x \in C$;

(h2) $F_{2}(x, \cdot)$ is monotone and upper semicontinuous;

(h3) $F_{2}(\cdot, x)$ is convex.

Moreover, let us suppose that

(H) for fixed $r>0$ and $x \in C$, there exists a bounded set $k \subset C$ and $a \in K$ such that for all $z \in C \backslash K,-F_{1}(a, z)+F_{2}(z, a)+\frac{1}{r}\langle a-z, z-x\rangle<0$, for $r>0$ and $x \in H$. Let $T_{r}: H \rightarrow C$ be a mapping defined by

$$
T_{r} x=\left\{z \in C: F_{1}(z, y)+F_{2}(z, y)+\frac{1}{r}\langle y-z, z-x\rangle \geq 0, \forall y \in C\right\},
$$

called a resolvent of $F_{1}$ and $F_{2}$.

Then

(i) $T_{r} x \neq \emptyset$;

(ii) $T_{r}$ is a single value;

(iii) $T_{r}$ is firmly nonexpansive;

(iv) $\operatorname{MEP}\left(F_{1}, F_{2}\right)=F_{i x}\left(T_{r}\right)$ and it is closed and convex.

Definition 2.9 Let $H$ be a real Hilbert space and $f: H \rightarrow H$ be a function.

(i) Minimization problem:

$$
\min _{x \in C} f(x)=\frac{1}{2}\left\|A x-P_{Q} A x\right\|^{2} .
$$

(ii) Tikhonov's regularization problem:

$$
\min _{x \in C} f_{\alpha}(x)=\frac{1}{2}\left\|A x-P_{Q} A x\right\|^{2}+\frac{1}{2} \alpha\|x\|^{2},
$$

where $\alpha>0$ is the regularization parameter.

Proposition 2.10 ([17]) If the SFP is consistent, then the strong $\lim _{\alpha \rightarrow 0} x_{\alpha}$ exists and is the minimum-norm solution of the SFP. 
Proposition 2.11 ([17]) A necessary and sufficient condition for $x_{\alpha}$ to converge in norm as $\alpha \rightarrow 0$ is that the minimization

$$
\lim _{u \in \overline{A(C)}} \operatorname{dist}(u, Q)=\min _{u \in \overline{A(C)}}\left\|u-P_{Q} u\right\|
$$

is attained at a point in the set $A(C)$.

Remark 2.12 ([17]) Assume that the SFP is consistent, and let $x_{\min }$ be its minimum-norm solution, namely $x_{\min } \in \Gamma$ has the property

$$
\left\|x_{\min }\right\|=\min \left\{\left\|x^{*}\right\|: x^{*} \in \Gamma\right\} .
$$

From (2.2), observing that the gradient

$$
\nabla f_{\alpha}(x)=\nabla f(x)+\alpha I=A^{*}\left(I-P_{Q}\right) A+\alpha I
$$

is an $\left(\alpha+\|A\|^{2}\right)$-Lipschitzian and $\alpha$-strongly monotone mapping, the mapping $P_{C}(I-$ $\left.\lambda \nabla f_{\alpha}\right)$ is a contraction with the coefficient

$$
\sqrt{1-\lambda\left(2 \alpha-\lambda\left(\|A\|^{2}+\alpha\right)^{2}\right)} \leq 1-\frac{1}{2} \alpha \lambda,
$$

where

$$
0<\lambda<\frac{\alpha}{\left(\|A\|^{2}+\alpha\right)^{2}} .
$$

Remark 2.13 The mapping $T=P_{C}\left(I-\lambda \nabla f_{\alpha}\right)$ is nonexpansive.

In fact, we have seen that $\nabla f=A^{*}\left(I-P_{Q}\right) A$ is $\frac{1}{\|A\|^{2}}$-inverse strongly monotone and $\lambda \nabla f=A^{\prime \prime}\left(I-P_{Q}\right) A$ is $\frac{1}{\lambda\|A\|^{2}}$-inverse strongly monotone, by Proposition 2.5(iii) the complement $I-\lambda \nabla f$ is $\frac{\lambda\|A\|^{2}}{2}$-averaged. Therefore, noting that $P_{C}$ is $\frac{1}{2}$-averaged and applying Proposition 2.6(iv), we know that for each $\lambda \in\left(0, \frac{2}{\lambda\|A\|^{2}}\right), T=P_{C}\left(I-\lambda \nabla f_{\alpha}\right)$ is $\alpha$-averaged, with

$$
\alpha=\frac{1}{2}+\frac{\lambda\|A\|^{2}}{2}-\frac{1}{2} \frac{\lambda\|A\|^{2}}{2}=\frac{2+\lambda\|A\|^{2}}{4} \in(0,1) .
$$

Hence, it is clear that $T$ is nonexpansive.

Lemma 2.14 ([17]) Assume that the SFP (1.1) is consistent. Define a sequence $\left\{x_{n}\right\}$ by the iterative algorithm

$$
x_{n+1}=P_{C}\left(I-\gamma_{n} \nabla f_{\alpha_{n}}\right) x_{n}=P_{C}\left(\left(1-\gamma_{n} \alpha_{n}\right) x_{n}-\gamma_{n} A^{*}\left(I-P_{Q}\right) A x_{n}\right),
$$

where $\left\{\alpha_{n}\right\}$ and $\left\{\gamma_{n}\right\}$ satisfy the following conditions:

(i) $0<\gamma_{n} \leq \frac{\alpha_{n}}{\|A\|^{2}+\alpha_{n}}$ for all $n$;

(ii) $\lim _{n \rightarrow \infty} \alpha_{n}=0$ and $\lim _{n \rightarrow \infty} \gamma_{n}=0$;

(iii) $\sum_{n=1}^{\infty} \alpha_{n} \gamma_{n}=\infty$;

(iv) $\lim _{n \rightarrow \infty} \frac{\left|\gamma_{n+1}-\gamma_{n}\right|-\gamma_{n}\left|\alpha_{n+1}-\alpha_{n}\right|}{\left(\alpha_{n+1} \gamma_{n+1}\right)^{2}}=0$.

Then $\left\{x_{n}\right\}$ converges in norm to the minimum-norm solution of the SFP (1.1). 
Lemma 2.15 ([18]) Let $\left\{x_{n}\right\}$ and $\left\{z_{n}\right\}$ be bounded sequences in a Banach space $X$ and let $\left\{\beta_{n}\right\}$ be a sequence in $[0,1] 0<\liminf _{n \rightarrow \infty} \beta_{n} \leq \lim \sup _{n \rightarrow \infty} \beta_{n}<1$. Suppose that $x_{n+1}=(1-$ $\left.\beta_{n}\right) z_{n}+\beta_{n} x_{n}$ for all $n \geq 0$ and $\lim \sup _{n \rightarrow \infty}\left(\left\|z_{n+1}-z_{n}\right\|-\left\|x_{n+1}-x_{n}\right\|\right) \leq 0$. Then, $\lim _{n \rightarrow \infty} \| z_{n}-$ $x_{n} \|=0$.

Lemma 2.16 ([19]) Let $K$ be a nonempty closed convex subset of a real Hilbert space $H$ and $T: K \rightarrow K$ be a nonexpansive mapping with $F_{i x}(T) \neq \emptyset$. If $\left\{x_{n}\right\}$ is a sequence in $K$ weakly converging to $x$ and if $\left\{(I-T) x_{n}\right\}$ converges strongly to $y$, then $(I-T) x=y$; in particular, if $y=0$, then $x \in F_{i x}(T)$.

Lemma 2.17 $([20,21])$ Assume $\left\{\alpha_{n}\right\}$ is a sequence of nonnegative real numbers such that

$$
\alpha_{n+1} \leq\left(1-\sigma_{n}\right) \alpha_{n}+\delta_{n} \sigma_{n}
$$

where $\left\{\sigma_{n}\right\}$ is a sequence in $(0,1)$ and $\left\{\delta_{n}\right\}$ is a sequence such that

(1) $\sum_{n=1}^{\infty} \sigma_{n}=\infty$,

(2) $\lim \sup _{n \rightarrow \infty} \delta_{n} \leq 0$ or $\sum_{n=1}^{\infty}\left|\delta_{n} \sigma_{n}\right|<\infty$.

Then $\lim _{n \rightarrow \infty} \alpha_{n}=0$.

\section{Main results}

In this section, we introduce two algorithms for solving the mixed equilibrium problem (1.3). Namely, we want to find a solution $x^{*}$ of the mixed equilibrium problem (1.3) and $x^{\prime \prime}$ also solves the following variational inequality:

$$
x^{*} \in \Gamma, \quad\left\langle(\gamma g-\mu B) x^{*}, x-x^{*}\right\rangle \leq 0, \quad x \in \Gamma,
$$

where $B$ is a $k$-Lipschitz and $\eta$-strongly monotone operator on $H$ with $k>0, \eta>0$ and $0<\mu<2 \eta / k^{2}$, and $g: C \rightarrow H$ is a $\beta$-contraction mapping, $\beta \in(0,1)$. Let $F_{1}, F_{2}: C \times C \rightarrow R$ be two bifunctions. In order to find a particular solution of the variational inequality (3.1), we construct the following implicit algorithm.

Algorithm 3.1 For an arbitrary initial point $x_{0}$, we define a sequence $\left\{x_{n}\right\}_{n \geq 0}$ iteratively

$$
x_{n}=(I-t \mu B) T_{r} P_{C}\left(I-\lambda_{n} \nabla f_{\alpha_{n}}\right) x_{n}, \quad \forall t \in(0,1),
$$

for all $n \geq 0$, where $\left\{\alpha_{n}\right\}$ is a real sequence in [0,1], $T_{r}$ is defined by Lemma 2.8 and $\nabla f_{\alpha_{n}}$ is introduced in Remark 2.12.

We show that the sequence $x_{n}$ defined by (3.2) converges to a particular solution of the variational inequality (3.1). As a matter of fact, in this paper, we study a general algorithm for solving the variational inequality (3.1).

Let $g: C \rightarrow H$ be a $\beta$-contraction mapping. For each $t \in(0,1)$, we consider the following mapping $S_{t}$ given by:

$$
S_{t} x=\left[t \gamma g+(I-t \mu B) T_{r} P_{C}\left(I-\lambda_{n} \nabla f_{\alpha_{n}}\right)\right] x, \quad x \in C .
$$


Lemma 3.2 $S_{t}$ is a contraction. Indeed,

$$
\left\|S_{t} x-S_{t} y\right\| \leq[1-(\tau-\gamma \beta) t]\|x-y\|, \quad \forall x, y \in H,
$$

where $t \in\left(0, \frac{1}{\tau-\gamma \beta}\right)$, and the sequence of $\left\{\alpha_{n}\right\}$ and $\left\{\gamma_{n}\right\}$ satisfy the conditions (i)-(iv) in Lemma 2.14.

Proof It is clear that $S_{t}$ is a self-mapping. Observe that

$$
\begin{aligned}
\| & \left(I-\lambda_{n} \nabla f_{\alpha_{n}}\right) x-\left(I-\lambda_{n} \nabla f_{\alpha_{n}}\right) y \|^{2} \\
\quad & \|x-y\|^{2}-2 \lambda_{n}\left(\nabla f_{\alpha_{n}}(x)-\nabla f_{\alpha_{n}}(y), x-y\right\rangle+\lambda_{n}^{2}\left\|\nabla f_{\alpha_{n}}(x)-\nabla f_{\alpha_{n}}(y)\right\|^{2} \\
& \leq\left(1-2 \lambda_{n} \alpha_{n}+\lambda_{n}^{2}\left(\alpha_{n}+\|A\|^{2}\right)^{2}\right)\|x-y\|^{2} \\
& =\left(1-\lambda_{n}\left(2 \alpha_{n}-\lambda_{n}\right)\left(\alpha_{n}+\|A\|^{2}\right)^{2}\right)\|x-y\|^{2} \\
& \leq\left(1-\lambda_{n} \alpha_{n}\right)\|x-y\|^{2} .
\end{aligned}
$$

Let $\mu\left(\eta-\frac{\mu k^{2}}{2}\right)=\tau$ and $t \in(0,1)$, we obtain

$$
\begin{aligned}
\|(I-t \mu B) x-(I-t \mu B) y\| & \leq\left[1-t \mu\left(\eta-\frac{\mu k^{2}}{2}\right)\right]\|x-y\| \\
& =[1-t \tau]\|x-y\| .
\end{aligned}
$$

Note that $P_{C}$ and $T_{r}$ are nonexpansive, $I-\lambda_{n} \nabla f_{\alpha_{n}}$ is a contraction mapping with the coefficient $1-\lambda \alpha_{n}$ and $\|I-t \mu B\| \leq 1-t \tau$. Hence, $\forall x, y \in C$, we obtain

$$
\begin{aligned}
\left\|S_{t} x-S_{t} y\right\|= & \|\left[t \gamma g+(I-t \mu B) T_{r} P_{C}\left(I-\lambda_{n} \nabla f_{\alpha_{n}}\right)\right] x \\
& -\left[t \gamma g+(I-t \mu B) T_{r} P_{C}\left(I-\lambda_{n} \nabla f_{\alpha_{n}}\right)\right] y \| \\
\leq & \|I-t \mu B\|\left\|\left(I-\lambda_{n} \nabla f_{\alpha_{n}}\right) x-\left(I-\lambda_{n} \nabla f_{\alpha_{n}}\right) y\right\| \\
& +t \gamma\|g(x)-g(y)\| \\
\leq & (1-t \tau)\left(1-\lambda_{n} \alpha_{n}\right)\|x-y\|^{2}+t \gamma \beta\|x-y\|^{2} \\
\leq & (1-(\tau-\gamma \beta) t)\|x-y\|^{2} .
\end{aligned}
$$

Therefore, $S_{t}$ is a contraction mapping when $t \in\left(0, \frac{1}{\tau-\gamma \beta}\right)$.

From Lemma 3.2 and using the Banach contraction principle, there exists a unique fixed point $x_{t}$ of $S_{t}$ in $C$, i.e., we obtain the following algorithm.

Algorithm 3.3 For an arbitrary initial point $x_{0}$, we define a sequence $\left\{x_{n}\right\}_{n \geq 0}$ iteratively

$$
x_{n}=\left[\varepsilon_{n} \gamma g+\left(I-\varepsilon_{n} \mu B\right) T_{r} P_{C}\left(I-\lambda_{n} \nabla f_{\alpha_{n}}\right)\right] x_{n}, \quad x \in C,
$$

for all $n \geq 0$, where $\left\{\alpha_{n}\right\}$ and $\left\{\varepsilon_{n}\right\}$ are two real sequences in [0,1], $T_{r}$ is defined by Lemma 2.8 and $\nabla f_{\alpha_{n}}$ is introduced in Remark 2.12.

At this point, we would like to point out that Algorithm 3.3 includes Algorithm 3.1 as a special case due to the fact that the contraction $g$ is a possible nonself-mapping. 
Theorem 3.4 Let $C$ be a nonempty closed convex subset of a real Hilbert space H. Let $B$ be a $k$-Lipschitz and $\eta$-strongly monotone operator on $H$ with $k>0, \eta>0$ and $0<\mu<2 \eta / k^{2}$, and the sequence of $\left\{\alpha_{n}\right\}$ and $\left\{\gamma_{n}\right\}$ satisfy the conditions (i)-(iv) in Lemma 2.14. Let $F_{1}, F_{2}$ : $C \times C \rightarrow R$ be two bifunctions which satisfy the conditions (f1)-(f4), (h1)-(h3) and (H) in Lemma 2.8. Let $g: C \rightarrow H$ be a $\beta$-contraction. Assume $\Omega:=\Gamma \cap \operatorname{MEP}\left(F_{1}, F_{2}\right) \neq \emptyset$. Then the sequence $\left\{x_{n}\right\}$ generated by implicit Algorithm 3.3 converges in norm, as $\varepsilon_{n} \rightarrow 0$, to the unique solution $x^{*}$ of the variational inequality (3.1). In particular, if we take $g=0$, then the sequence $\left\{x_{n}\right\}$ defined by Algorithm 3.1 converges in norm, as $\varepsilon_{n} \rightarrow 0$, to the unique solution $x^{*}$ of the following variational inequality:

$$
\left\langle\mu B x^{*}, x-x^{*}\right| \geq 0, \quad \forall x \in \Omega \text {. }
$$

Proof Next, we divide the remainder of the proof into several steps.

Step 1 . We prove that the sequence $\left\{x_{n}\right\}$ is bounded.

Set $u_{n}=P_{C}\left(I-\lambda_{n} \nabla f_{\alpha_{n}}\right) x_{n}$ for all $n \geq 0$. Take $q \in \Omega$. It is clear that $q=P_{C}\left(I-\lambda_{n} \nabla f_{\alpha_{n}}\right) q$. From Remark 2.13, we know that $P_{C}\left(I-\lambda_{n} \nabla f_{\alpha_{n}}\right)$ is nonexpansive, then we have

$$
\left\|u_{n}-q\right\|=\left\|P_{C}\left(I-\lambda_{n} \nabla f_{\alpha_{n}}\right) x_{n}-P_{C}\left(I-\lambda_{n} \nabla f_{\alpha_{n}}\right) q\right\| \leq\left\|x_{n}-q\right\| .
$$

From (3.5), (3.6) and the fact that $T_{r}$ is nonexpansive, it follows that

$$
\begin{aligned}
\left\|x_{n}-q\right\| & =\left\|\left[\varepsilon_{n} \gamma g\left(x_{n}\right)+\left(I-\varepsilon_{n} \mu B\right) T_{r} u_{n}\right]-P_{C} q\right\| \\
& =\left\|\varepsilon_{n} \gamma\left(g\left(x_{n}\right)-g(q)\right)+\left(I-\varepsilon_{n} \mu B\right)\left(T_{r} u_{n}-q\right)+\varepsilon_{n}(\gamma g(q)-\mu B q)\right\| \\
& \leq \varepsilon_{n} \gamma \beta\left\|x_{n}-q\right\|+\left(1-\varepsilon_{n} \tau\right)\left\|T_{r} u_{n}-q\right\|+\varepsilon_{n}\|\mu B q-\gamma g(q)\| \\
& \leq\left(1-\varepsilon_{n}(\tau-\gamma \beta)\right)\left\|x_{n}-q\right\|+\varepsilon_{n}\|\mu B q-\gamma g(q)\| .
\end{aligned}
$$

It follows by induction that

$$
\begin{aligned}
\left\|x_{n}-q\right\| & \leq \max \left\{\left\|x_{n}-q\right\|,\|\mu B q-\gamma g(q)\| /(\tau-\gamma \beta)\right\} \\
& \leq \max \left\{\left\|x_{0}-q\right\|,\|\mu B q-\gamma g(q)\| /(\tau-\gamma \beta)\right\} .
\end{aligned}
$$

This indicates that $\left\{x_{n}\right\}$ is bounded. It is easy to deduce that $\left\{g\left(x_{n}\right)\right\}$ and $\left\{u_{n}\right\}$ are also bounded.

Now, we can choose a constant $M>0$ such that

$$
\begin{aligned}
& \sup _{n}\left\{\left\|x_{n}-u_{n}\right\|,\left\|\gamma g\left(x_{n}\right)-\mu B q\right\|\left\|T_{r} u_{n}-q\right\|+\left\|\gamma g\left(x_{n}\right)-\mu B q\right\|^{2},\right. \\
& \left.\left\|\gamma g\left(x_{n}\right)-\mu B T_{r} u_{n}\right\|^{2}\right\} \leq M .
\end{aligned}
$$

Step 2. We prove that $\lim _{n \rightarrow \infty}\left\|x_{n}-u_{n}\right\|=0$.

From (3.5), (3.6) and the fact that $T_{r}$ is nonexpansive, we have

$$
\begin{aligned}
\left\|x_{n}-q\right\|^{2} & =\left\|\left(I-\varepsilon_{n} \mu B\right)\left(T_{r} u_{n}-q\right)+\varepsilon_{n}\left(\gamma g\left(x_{n}\right)-\mu B q\right)\right\|^{2} \\
& \leq\left(1-\varepsilon_{n} \tau\right)\left\|T_{r} u_{n}-q\right\|^{2}+\varepsilon_{n}\left(1-\varepsilon_{n} \tau\right)\left\langle\left(\gamma g\left(x_{n}\right)-\mu B q\right), T_{r} u_{n}-q\right\rangle
\end{aligned}
$$




$$
\begin{aligned}
& +\varepsilon_{n}\left\|\gamma g\left(x_{n}\right)-\mu B q\right\|^{2} \\
\leq & \left(1-\varepsilon_{n} \tau\right)\left\|T_{r} u_{n}-q\right\|^{2}+\varepsilon_{n}\left\|\gamma g\left(x_{n}\right)-\mu B q\right\|\left\|T_{r} u_{n}-q\right\| \\
& +\varepsilon_{n}\left\|\gamma g\left(x_{n}\right)-\mu B q\right\|^{2} \\
\leq & \left(1-\varepsilon_{n} \tau\right)\left\|u_{n}-q\right\|^{2}+\varepsilon_{n} M .
\end{aligned}
$$

Note that $\nabla f_{\alpha_{n}}(x)$ is an $\left(\alpha+\|A\|^{2}\right)$-Lipschitzian and $\alpha$-strongly monotone mapping. From Lemma 2.8, (3.5) and (3.6), we have

$$
\begin{aligned}
\left\|u_{n}-q\right\|^{2}= & \left\|P_{C}\left(I-\lambda_{n} \nabla f_{\alpha_{n}}\right) x_{n}-P_{C}\left(I-\lambda_{n} \nabla f_{\alpha_{n}}\right) q\right\|^{2} \\
\leq & \left\langle\left(I-\lambda_{n} \nabla f_{\alpha_{n}}\right) x_{n}-\left(I-\lambda_{n} \nabla f_{\alpha_{n}}\right) q, u_{n}-q\right\rangle \\
= & \frac{1}{2}\left(\left\|\left(I-\lambda_{n} \nabla f_{\alpha_{n}}\right) x_{n}-\left(I-\lambda_{n} \nabla f_{\alpha_{n}}\right) q\right\|^{2}\right. \\
& \left.+\left\|u_{n}-q\right\|^{2}-\left\|x_{n}-u_{n}-\lambda_{n}\left(\nabla f_{\alpha_{n}}\left(x_{n}\right)-\nabla f_{\alpha_{n}}(q)\right)\right\|^{2}\right) \\
\leq & \frac{1}{2}\left(\left(1-\lambda_{n} \alpha_{n}\right)\left\|x_{n}-q\right\|^{2}+\left\|u_{n}-q\right\|^{2}\right. \\
& \left.-\left\|x_{n}-u_{n}-\lambda_{n}\left(\nabla f_{\alpha_{n}}\left(x_{n}\right)-\nabla f_{\alpha_{n}}(q)\right)\right\|^{2}\right) \\
\leq & \frac{1}{2}\left(\left\|x_{n}-q\right\|^{2}+\left\|u_{n}-q\right\|^{2}-\left\|x_{n}-u_{n}\right\|^{2}\right. \\
& \left.+2 \lambda_{n}\left(x_{n}-u_{n}, \nabla f_{\alpha_{n}}\left(x_{n}\right)-\nabla f_{\alpha_{n}}(q)\right\rangle-\lambda_{n}^{2}\left\|\nabla f_{\alpha_{n}}\left(x_{n}\right)-\nabla f_{\alpha_{n}}(q)\right\|^{2}\right),
\end{aligned}
$$

which implies that

$$
\begin{aligned}
\left\|u_{n}-q\right\|^{2} \leq & \left\|x_{n}-q\right\|^{2}-\left\|x_{n}-u_{n}\right\|^{2} \\
& +2 \lambda_{n}\left(x_{n}-u_{n}, \nabla f_{\alpha_{n}}\left(x_{n}\right)-\nabla f_{\alpha_{n}}(q)\right\rangle-\lambda_{n}^{2}\left\|\nabla f_{\alpha_{n}}\left(x_{n}\right)-\nabla f_{\alpha_{n}}(q)\right\|^{2} \\
\leq & \left\|x_{n}-q\right\|^{2}-\left\|x_{n}-u_{n}\right\|^{2}+2 \lambda_{n}\left\|x_{n}-u_{n}\right\|\left\|\nabla f_{\alpha_{n}}\left(x_{n}\right)-\nabla f_{\alpha_{n}}(q)\right\| .
\end{aligned}
$$

By (3.7) and (3.8), we obtain

$$
\left\|u_{n}-q\right\|^{2} \leq\left(1-\varepsilon_{n} \tau\right)\left\|u_{n}-q\right\|^{2}+\varepsilon_{n} M-\left\|x_{n}-u_{n}\right\|^{2}+2 \lambda_{n}\left\|x_{n}-u_{n}\right\|\left\|\nabla f_{\alpha_{n}}\left(x_{n}\right)-\nabla f_{\alpha_{n}}(q)\right\| .
$$

It follows that

$$
\left\|x_{n}-u_{n}\right\|^{2} \leq \varepsilon_{n} M+2 \lambda_{n}\left\|x_{n}-u_{n}\right\|\left\|\nabla f_{\alpha_{n}}\left(x_{n}\right)-\nabla f_{\alpha_{n}}(q)\right\| .
$$

This together with $\lim _{n \rightarrow \infty} \varepsilon_{n}=0$ and $\lim _{n \rightarrow \infty} \lambda_{n}=0$ implies that

$$
\lim _{n \rightarrow \infty}\left\|x_{n}-u_{n}\right\|=0
$$

Setting $y_{n}=T_{r} u_{n}$, we have

$$
\begin{aligned}
\left\|x_{n}-y_{n}\right\| & =\left\|\varepsilon_{n} \gamma g\left(x_{n}\right)+\left(I-\varepsilon_{n} \mu B\right) y_{n}-y_{n}\right\| \\
& \leq\left\|\varepsilon_{n} \gamma g\left(x_{n}\right)-\varepsilon_{n} \mu B T_{r} u_{n}\right\|
\end{aligned}
$$




$$
\begin{aligned}
& \leq \varepsilon_{n}\left\|\gamma g\left(x_{n}\right)-\gamma g\left(u_{n}\right)\right\|+\varepsilon_{n}\left\|\gamma g\left(u_{n}\right)-\mu B T_{r} u_{n}\right\| \\
& \leq \varepsilon_{n} \gamma \beta\left\|x_{n}-u_{n}\right\|+\varepsilon_{n}\left\|\gamma g\left(u_{n}\right)-\mu B T_{r} u_{n}\right\| .
\end{aligned}
$$

From $\lim _{n \rightarrow \infty} \varepsilon_{n}=0,\left\{u_{n}\right\}$ is bounded and (3.9), we obtain

$$
\lim _{n \rightarrow \infty}\left\|x_{n}-y_{n}\right\|=0 .
$$

By (3.9) and (3.10), we also have

$$
\lim _{n \rightarrow \infty}\left\|u_{n}-y_{n}\right\|=\lim _{n \rightarrow \infty}\left(\left\|u_{n}-x_{n}\right\|-\left\|x_{n}-y_{n}\right\|\right)=0
$$

Step 3. We prove $u_{n} \rightarrow x^{*} \in \Omega:=\Gamma \cap \operatorname{MEP}\left(F_{1}, F_{2}\right)$.

By (3.4) and (3.5), we deduce

$$
\begin{aligned}
\left\|x_{n}-x^{*}\right\|^{2}= & \left\|\left[\varepsilon_{n} \gamma g\left(x_{n}\right)+\left(I-\varepsilon_{n} \mu B\right) y_{n}\right]-x^{*}\right\|^{2} \\
\leq & \left\|y_{n}-x^{*}+\varepsilon_{n} \gamma g\left(x_{n}\right)-\varepsilon_{n} \mu B y_{n}\right\|^{2} \\
= & \left\|T_{r} u_{n}-x^{*}\right\|^{2}+2 \varepsilon_{n} \gamma\left\langle g\left(x_{n}\right), y_{n}-x^{*}\right\rangle-2 \varepsilon_{n}\left\langle\mu B y_{n}, y_{n}-x^{*}\right\rangle \\
& +\varepsilon_{n}^{2}\left\|\gamma g\left(x_{n}\right)-\mu B y_{n}\right\|^{2} \\
= & \left\|u_{n}-x^{*}\right\|^{2}+2 \varepsilon_{n}\left\langle\gamma g\left(x_{n}\right)-\gamma g\left(x^{*}\right), y_{n}-x^{*}\right\rangle+2 \varepsilon_{n}\left\langle\gamma g\left(x^{*}\right)-\mu B x^{*}, y_{n}-x^{*}\right\rangle \\
& -2 \varepsilon_{n}\left\langle\mu B T_{r} u_{n}-\mu B x^{*}, y_{n}-x^{*}\right\rangle+\varepsilon_{n}^{2}\left\|\gamma g\left(x_{n}\right)-\mu B y_{n}\right\|^{2} \\
\leq & \left\|u_{n}-x^{*}\right\|^{2}+2 \varepsilon_{n} \gamma \beta\left\|x_{n}-x^{*}\right\|\left\|u_{n}-x^{*}\right\|+2 \varepsilon_{n}\left\langle\gamma g\left(x^{*}\right)-\mu B x^{*}, y_{n}-x^{*}\right\rangle \\
& -2 \varepsilon_{n} \tau\left\|u_{n}-x^{*}\right\|^{2}+\varepsilon_{n}^{2}\left\|\gamma g\left(x_{n}\right)-\mu B y_{n}\right\|^{2} \\
\leq & \left(1-2 \varepsilon_{n} \tau\right)\left(1-\lambda_{n} \alpha_{n}\right)\left\|x_{n}-x^{*}\right\|^{2}+2 \varepsilon_{n} \gamma \beta\left(1-\lambda_{n} \alpha_{n}\right)\left\|x_{n}-x^{*}\right\|^{2} \\
& +2 \varepsilon_{n}\left\langle\gamma g\left(x^{*}\right)-\mu B x^{*}, y_{n}-x^{*}\right\rangle+\varepsilon_{n}^{2}\left\|\gamma g\left(x_{n}\right)-\mu B y_{n}\right\|^{2} \\
\leq & \left(1-2 \varepsilon_{n}(\tau-\gamma \beta)\right)\left(1-\lambda_{n} \alpha_{n}\right)\left\|x_{n}-x^{*}\right\|^{2}+2 \varepsilon_{n}\left\langle\gamma g\left(x^{*}\right)-\mu B x^{*}, y_{n}-x^{*}\right\rangle \\
& +\varepsilon_{n}^{2}\left\|\gamma g\left(x_{n}\right)-\mu B y_{n}\right\|^{2} \\
\leq & \left(1-2 \varepsilon_{n}(\tau-\gamma \beta)\right)\left\|x_{n}-x^{*}\right\|^{2}+2 \varepsilon_{n}\left\langle\gamma g\left(x^{*}\right)-\mu B x^{*}, y_{n}-x^{*}\right\rangle \\
& +\varepsilon_{n}^{2}\left\|\gamma g\left(x_{n}\right)-\mu B y_{n}\right\|^{2} .
\end{aligned}
$$

It follows that

$$
\begin{aligned}
\left\|x_{n}-x^{*}\right\|^{2} & \leq \frac{1}{\tau-\gamma \beta}\left\langle\gamma g\left(x^{*}\right)-\mu B x^{*}, y_{n}-x^{*}\right\rangle+\frac{\varepsilon_{n}}{2(\tau-\gamma \beta)}\left\|\gamma g\left(x_{n}\right)-\mu B y_{n}\right\|^{2} \\
& \leq \frac{1}{\tau-\gamma \beta}\left\langle\gamma g\left(x^{*}\right)-\mu B x^{*}, y_{n}-x^{*}\right\rangle+\frac{\varepsilon_{n}}{2(\tau-\gamma \beta)} M .
\end{aligned}
$$

Since $\left\{x_{n}\right\}$ is bounded, without loss of generality, we may assume that $\left\{x_{n}\right\}$ converges weakly to a point $x^{*} \in C$. Hence, $u_{n} \rightarrow x^{*}$ and $y_{n} \rightarrow x^{*}$.

Step 4. We show $x^{\prime \prime} \in \omega_{w}\left(x_{n}\right) \subset \Omega:=\Gamma \cap \operatorname{MEP}\left(F_{1}, F_{2}\right)$.

Since $y_{n}=T_{r} u_{n}$, for any $y \in C$, we obtain

$$
F_{1}\left(y_{n}-y\right)+F_{2}\left(y_{n}-y\right)+\frac{1}{r}\left\langle y-y_{n}, y_{n}-u_{n}\right\rangle \geq 0 .
$$


From the monotonicity of $F_{1}$ and $F_{2}$, we get

$$
\frac{1}{r}\left\langle y-y_{n}, y_{n}-u_{n}\right\rangle \geq F_{1}\left(y-y_{n}\right)+F_{2}\left(y-y_{n}\right), \quad \forall y \in C
$$

Hence,

$$
\left\langle y-y_{n_{i}}, \frac{y_{n_{i}}-x_{n_{i}}}{r}\right\rangle \geq F_{1}\left(y-y_{n_{i}}\right)+F_{2}\left(y-y_{n_{i}}\right), \quad \forall y \in C .
$$

Since $\frac{y_{n_{i}}-x_{n_{i}}}{r} \rightarrow 0$ and $y_{n} \rightarrow x^{*}$, from (A4), it follows $F_{1}\left(y-x^{*}\right)+F_{2}\left(y-x^{*}\right) \leq 0$ for all $y \in H$. Put $z_{t}=t y+(1-t) x^{*}$ for all $t \in(0,1]$ and $y \in H$, then we have $F_{1}\left(z_{t}-x^{*}\right)+F_{2}\left(z_{t}-x^{*}\right) \leq 0$, So, from (A1) and (A4), we have

$$
\begin{aligned}
0 & =F_{1}\left(y_{t}, y_{t}\right)+F_{2}\left(y_{t}, y_{t}\right) \\
& \leq t F_{1}\left(y_{t}, y\right)+(1-t) F_{1}\left(y_{t}, x^{*}\right)+t F_{2}\left(y_{t}, y\right)+(1-t) F_{2}\left(y_{t}, x^{*}\right) \\
& \leq F_{1}\left(y_{t}, y\right)+F_{2}\left(y_{t}, y\right)
\end{aligned}
$$

and hence $0 \leq F_{1}\left(y_{t}, y\right)+F_{2}\left(y_{t}, y\right)$. From (A3), we have $0 \leq F_{1}\left(x^{*}, y\right)+F_{2}\left(x^{*}, y\right)$ for all $y \in H$. Therefore, $x^{*} \in \operatorname{MEP}\left(F_{1}, F_{2}\right)$.

Next, we prove $x^{*} \in \Gamma$.

From Remark 2.13, we know that $T=P_{C}\left(I-\lambda_{n} \nabla f\right)$ is nonexpansive, then we have

$$
\begin{aligned}
\left\|x_{n}-T x_{n}\right\| & \leq\left\|x_{n}-u_{n}\right\|+\left\|u_{n}-T x_{n}\right\| \\
& =\left\|x_{n}-u_{n}\right\|+\left\|P_{C}\left(I-\lambda_{n} \nabla f_{\alpha_{n}}\right) x_{n}-P_{C}\left(I-\lambda_{n} \nabla f\right) x_{n}\right\| \\
& =\left\|x_{n}-u_{n}\right\|+\left\|\left(I-\lambda_{n} \nabla f_{\alpha_{n}}\right) x_{n}-\left(I-\lambda_{n} \nabla f\right) x_{n}\right\| \\
& =\left\|x_{n}-u_{n}\right\|+\lambda_{n} \alpha_{n}\left\|x_{n}\right\| .
\end{aligned}
$$

So, from $\lim _{n \rightarrow \infty}\left\|x_{n}-u_{n}\right\|=0, \lim _{n \rightarrow \infty} \alpha_{n}=0, \lim _{n \rightarrow \infty} \lambda_{n}=0, \sum_{n=1}^{\infty} \alpha_{n} \lambda_{n}=\infty$ and the bounded sequence of $\left\{x_{n}\right\}$ it follows that

$$
\lim _{n \rightarrow \infty}\left\|x_{n}-T x_{n}\right\|=0
$$

Thus, taking into account $x_{n_{j}} \rightarrow x^{*}$ and $u_{n_{j}} \rightarrow x^{*}$, and from Lemma 2.16, we get $x^{*} \in \Gamma$. Therefore, we have $x^{*} \in \Omega:=\Gamma \cap \operatorname{MEP}\left(F_{1}, F_{2}\right)$. This shows that it holds that

$$
\omega_{w}\left(x_{n}\right) \subset \Omega:=\Gamma \cap \operatorname{MEP}\left(F_{1}, F_{2}\right) .
$$

Step 5. $\lim _{n \rightarrow \infty} x_{n}=x^{*}$.

We substitute $x^{*}$ for $z$ in (3.12) to get

$$
\left\|x_{n}-x^{*}\right\|^{2} \leq \frac{1}{\tau-\gamma \beta}\left\langle\gamma g\left(x^{*}\right)-\mu B x^{*}, y_{n}-x^{*}\right\rangle+\frac{\varepsilon_{n}}{2(\tau-\gamma \beta)} M .
$$

Hence, the weak convergence of $y_{n} \rightarrow x^{*}$ implies that $x_{n} \rightarrow x^{*}$ strongly. 
Now, we return to (3.12) and take the limit as $n \rightarrow \infty$ to obtain

$$
\left\|x^{*}-z\right\|^{2} \leq \frac{1}{\tau-\gamma \beta}\left\langle\mu B z-\gamma g(z), z-x^{*}\right\rangle, \quad z \in \Omega .
$$

In particular, $x^{*}$ solves the following variational inequality:

$$
z \in \Omega, \quad\left\langle\mu B z-\gamma g(z), z-x^{*}\right| \geq 0, \quad x^{*} \in \Omega,
$$

or the equivalent dual variational inequality

$$
z \in \Omega, \quad\left\langle\mu B x^{*}-\gamma g\left(x^{*}\right), z-x^{*}\right| \geq 0, \quad x^{*} \in \Omega .
$$

Therefore, $x^{*}=\left(P_{\Omega} g\right) x^{*}$. That is, $x^{*}$ is the unique fixed point in $\Omega$ of the contraction $P_{\Omega} g$.

Remark 3.5 If we take $g=0$, then (3.15) is reduced to

$$
\left\|x^{*}-z\right\|^{2} \leq\left\langle\mu B z, z-x^{*}\right\rangle, \quad z \in \Omega
$$

Equivalently,

$$
\left\|x^{*}\right\|^{2} \leq\left\langle z, x^{*}\right\rangle, \quad z \in \Omega
$$

This clearly implies that

$$
\left\|x^{*}\right\|^{2} \leq\|z\|, \quad z \in \Omega \text {. }
$$

Therefore, $x^{*}$ is a particular solution of the variational inequality (3.1).

Next, we introduce an explicit algorithm for finding a solution of the variational inequality (3.1). This scheme is obtained by discretizing the implicit scheme (3.3). We show the strong convergence of this algorithm.

Theorem 3.6 Let $C$ be a nonempty closed convex subset of a real Hilbert space H. Let $B$ be a $k$-Lipschitz and $\eta$-strongly monotone operator on $H$ with $k>0, \eta>0$ and $0<\mu<2 \eta / k^{2}$, and the sequence of $\left\{\alpha_{n}\right\}$ and $\left\{\gamma_{n}\right\}$ satisfy the conditions (i)-(iv) in Lemma 2.14. Let $F_{1}, F_{2}$ : $C \times C \rightarrow R$ be two bifunctions which satisfy the conditions (f1)-(f4), (h1)-(h3) and (H) in Lemma 2.8. Let $g: C \rightarrow H$ be a $\beta$-contraction. Assume $\Omega:=\Gamma \cap \operatorname{MEP}\left(F_{1}, F_{2}\right) \neq \emptyset$. For given $\forall x_{0} \in C$, let the sequence $\left\{x_{n}\right\}$ generated by

$$
x_{n+1}=\theta_{n} x_{n}+\left(1-\theta_{n}\right)\left[\varepsilon_{n} \gamma g+\left(I-\varepsilon_{n} \mu B\right) T_{r} P_{C}\left(I-\lambda_{n} \nabla f_{\alpha_{n}}\right)\right] x_{n}, \quad n \geq 0,
$$

where $\left\{\varepsilon_{n}\right\}$ and $\left\{\theta_{n}\right\}$ are two sequences in $[0,1]$, satisfy the following conditions:

(i) $\lim _{n \rightarrow \infty} \varepsilon_{n}=0$ and $\sum_{n=1}^{\infty} \varepsilon_{n}=\infty$;

(ii) $0<\liminf _{n \rightarrow \infty} \theta_{n} \leq \limsup _{n \rightarrow \infty} \theta_{n}<1$;

(iii) $\lim _{n \rightarrow \infty} \lambda_{n}=0$. 
Then the sequence $\left\{x_{n}\right\}$ converges strongly to $x^{*}$ which is the unique solution of the variational inequality (3.1). In particular, if $g=0$, then the sequence $\left\{x_{n}\right\}$ generated by

$$
x_{n+1}=\theta_{n} x_{n}+\left(1-\theta_{n}\right)\left[\left(I-\varepsilon_{n} \mu B\right) T_{r} P_{C}\left(I-\lambda_{n} \nabla f_{\alpha_{n}}\right)\right] x_{n}, \quad n \geq 0,
$$

converges strongly to a solution of the following variational inequality:

$$
\left\langle\mu B x^{*}, x-x^{*}\right\rangle \geq 0, \quad \forall x \in \Omega .
$$

Proof First, we prove that the sequence $\left\{x_{n}\right\}$ is bounded. Indeed, pick $z \in \Omega$.

Let $z=P_{C}\left(I-\lambda_{n} \nabla f_{\alpha_{n}}\right) z$. Set $u_{n}=P_{C}\left(I-\lambda_{n} \nabla f_{\alpha_{n}}\right) x_{n}$ for all $n \geq 0$. From (3.16), we have

$$
\left\|u_{n}-z\right\|=\left\|P_{C}\left(I-\lambda_{n} \nabla f_{\alpha_{n}}\right) x_{n}-P_{C}\left(\left(I-\lambda_{n} \nabla f_{\alpha_{n}}\right)\right) z\right\| \leq\left\|x_{n}-z\right\|,
$$

and

$$
\begin{aligned}
\left\|x_{n+1}-z\right\|= & \left\|\theta_{n} x_{n}+\left(1-\theta_{n}\right)\left[\varepsilon_{n} \gamma g\left(x_{n}\right)+\left(I-\varepsilon_{n} \mu B\right) T_{r} u_{n}\right]-z\right\| \\
\leq & \theta_{n}\left\|x_{n}-z\right\|+\left(1-\theta_{n}\right)\left\|\left(I-\varepsilon_{n} \mu B\right)\left(T_{r} u_{n}-z\right)+\varepsilon_{n}\left(\gamma g\left(x_{n}\right)-\mu B z\right)\right\| \\
\leq & \theta_{n}\left\|x_{n}-z\right\|+\left(1-\theta_{n}\right)\left[\left(1-\varepsilon_{n} \tau\right)\left\|u_{n}-z\right\|+\varepsilon_{n} \gamma \beta\left\|x_{n}-z\right\|\right. \\
& \left.+\varepsilon_{n}\|\gamma g(z)-\mu B z\|\right] \\
\leq & \theta_{n}\left\|x_{n}-z\right\|+\left(1-\theta_{n}\right)\left[\left(1-\varepsilon_{n} \tau\right)\left\|x_{n}-z\right\|+\varepsilon_{n} \gamma \beta\left\|x_{n}-z\right\|\right. \\
& \left.+\varepsilon_{n}\|\gamma g(z)-\mu B z\|\right] \\
= & {\left[1-(\tau-\gamma \beta)\left(1-\theta_{n}\right) \varepsilon_{n}\right]\left\|x_{n}-z\right\|+\varepsilon_{n}\left(1-\theta_{n}\right)\|\gamma g(z)-\mu B z\| } \\
\leq & \max \left\{\left\|x_{n}-z\right\|, \frac{\|\gamma g(z)-\mu B z\|}{\tau-\gamma \beta}\right\} .
\end{aligned}
$$

By induction, we have, $\forall n>0$,

$$
\left\|x_{n}-z\right\| \leq \max \left\{\left\|x_{0}-z\right\|, \frac{\|\gamma g(z)-\mu B z\|}{\tau-\gamma \beta}\right\} .
$$

Hence, $\left\{x_{n}\right\}$ is bounded. Consequently, we deduce that $\left\{u_{n}\right\},\left\{g\left(x_{n}\right)\right\}$ and $\left\{\nabla f\left(x_{n}\right)\right\}$ are all bounded. Let $M>0$ be a constant such that

$$
\sup _{n}\left\{\left\|x_{n}-u_{n}\right\|,\left\|\mu B T_{r} u_{n}\right\|+\left\|\gamma g\left(x_{n}\right)\right\|,\left\|\mu B T_{r} u_{n}-\gamma g\left(x_{n}\right)\right\|^{2}\right\} \leq M .
$$

Next, we show $\lim _{n \rightarrow \infty}\left\|x_{n}-u_{n}\right\|=0$.

Define $x_{n+1}=\theta_{n} x_{n}+\left(1-\theta_{n}\right) v_{n}$ for all $n>0$. It follows from (3.16) that

$$
\begin{aligned}
& \left\|v_{n+1}-v_{n}\right\| \\
& \quad=\left\|\left[\varepsilon_{n+1} \gamma g\left(x_{n+1}\right)+\left(I-\varepsilon_{n+1} \mu B\right) T_{r} u_{n+1}\right]-\left[\varepsilon_{n} \gamma g\left(x_{n}\right)+\left(I-\varepsilon_{n} \mu B\right) T_{r} u_{n}\right]\right\| \\
& \quad \leq\left\|u_{n+1}-u_{n}\right\|+\varepsilon_{n+1}\left(\left\|\mu B T_{r} u_{n+1}\right\|+\left\|\gamma g\left(x_{n+1}\right)\right\|\right)+\varepsilon_{n}\left(\left\|\mu B T_{r} u_{n}\right\|+\left\|\gamma g\left(x_{n}\right)\right\|\right) \\
& \quad \leq\left\|P_{C}\left(I-\lambda_{n} \nabla f_{\alpha_{n}}\right) x_{n+1}-P_{C}\left(I-\lambda_{n} \nabla f_{\alpha_{n}}\right) x_{n}\right\|+M\left(\varepsilon_{n+1}+\varepsilon_{n}\right) \\
& \quad \leq\left\|x_{n+1}-x_{n}\right\|+M\left(\varepsilon_{n+1}+\varepsilon_{n}\right) .
\end{aligned}
$$


This together with (i) implies that

$$
\limsup _{n \rightarrow \infty}\left(\left\|v_{n+1}-v_{n}\right\|-\left\|x_{n+1}-x_{n}\right\|\right) \leq 0
$$

Hence, by Lemma 2.15, we get $\lim _{n \rightarrow \infty}\left\|v_{n}-x_{n}\right\|=0$. Consequently,

$$
\lim _{n \rightarrow \infty}\left\|x_{n+1}-x_{n}\right\|=\lim _{n \rightarrow \infty}\left(1-\theta_{n}\right)\left\|v_{n}-x_{n}\right\|=0
$$

By the convexity of the norm $\|\cdot\|$, we obtain

$$
\begin{aligned}
\left\|x_{n+1}-z\right\|^{2}= & \left\|\theta_{n} x_{n}+\left(1-\theta_{n}\right) v_{n}-z\right\|^{2} \\
\leq & \theta_{n}\left\|x_{n}-z\right\|^{2}+\left(1-\theta_{n}\right)\left\|v_{n}-z\right\|^{2} \\
\leq & \theta_{n}\left\|x_{n}-z\right\|^{2}+\left(1-\theta_{n}\right)\left\|T_{r} u_{n}-z-\varepsilon_{n}\left(\mu B T_{r} u_{n}-\gamma g\left(x_{n}\right)\right)\right\|^{2} \\
= & \theta_{n}\left\|x_{n}-z\right\|^{2}+\left(1-\theta_{n}\right)\left[\left\|u_{n}-z\right\|^{2}\right. \\
& \left.-2 \varepsilon_{n}\left\langle\mu B T_{r} u_{n}-\gamma g\left(x_{n}\right), T_{r} u_{n}-z\right\rangle+\varepsilon_{n}^{2}\left\|\mu B T_{r} u_{n}-\gamma g\left(x_{n}\right)\right\|^{2}\right] \\
\leq & \theta_{n}\left\|x_{n}-z\right\|^{2}+\left(1-\theta_{n}\right)\left\|u_{n}-z\right\|^{2}+\varepsilon_{n} M .
\end{aligned}
$$

Let $y_{n}=T_{r} u_{n}$ and by $u_{n}=P_{C}\left(I-\lambda_{n} \nabla f_{\alpha_{n}}\right) x_{n}$, we obtain

$$
\begin{aligned}
\left\|y_{n}-z\right\|^{2}= & \left\|T_{r} u_{n}-T_{r} z\right\|^{2} \\
\leq & \left\|u_{n}-z\right\|^{2} \\
= & \left\|P_{C}\left(I-\lambda_{n} \nabla f_{\alpha_{n}}\right) x_{n}-P_{C}\left(I-\lambda_{n} \nabla f_{\alpha_{n}}\right) z\right\|^{2} \\
\leq & \left\langle\left(I-\lambda_{n} \nabla f_{\alpha_{n}}\right) x_{n}-\left(I-\lambda_{n} \nabla f_{\alpha_{n}}\right) z, u_{n}-z\right\rangle \\
= & \frac{1}{2}\left(\left\|\left(I-\lambda_{n} \nabla f_{\alpha_{n}}\right) x_{n}-\left(I-\lambda_{n} \nabla f_{\alpha_{n}}\right) z\right\|^{2}+\left\|u_{n}-z\right\|^{2}\right. \\
& \left.-\left\|\left(x_{n}-z\right)-\lambda_{n}\left(\nabla f_{\alpha_{n}}\left(x_{n}\right)-\nabla f_{\alpha_{n}}(z)\right)-\left(u_{n}-z\right)\right\|^{2}\right) \\
\leq & \frac{1}{2}\left(\left\|x_{n}-z\right\|^{2}+\left\|u_{n}-z\right\|^{2}-\left\|\left(x_{n}-u_{n}\right)-\lambda_{n}\left(\nabla f_{\alpha_{n}}\left(x_{n}\right)-\nabla f_{\alpha_{n}}(z)\right)\right\|^{2}\right) \\
\leq & \frac{1}{2}\left(\left\|x_{n}-z\right\|^{2}+\left\|u_{n}-z\right\|^{2}-\left\|x_{n}-u_{n}\right\|^{2}+2 \lambda_{n}\left\langle x_{n}-u_{n}, \nabla f_{\alpha_{n}}\left(x_{n}\right)-\nabla f_{\alpha_{n}}(z)\right\rangle\right. \\
& \left.-\lambda_{n}^{2}\left\|\nabla f_{\alpha_{n}}\left(x_{n}\right)-\nabla f_{\alpha_{n}}(z)\right\|^{2}\right) .
\end{aligned}
$$

Thus, we deduce

$$
\begin{aligned}
\left\|u_{n}-z\right\|^{2} & \leq\left\|x_{n}-z\right\|^{2}-\left\|x_{n}-u_{n}\right\|^{2}+2 \lambda_{n}\left\|x_{n}-u_{n}\right\|\left\|\nabla f_{\alpha_{n}}\left(x_{n}\right)-\nabla f_{\alpha_{n}}(z)\right\| \\
& \leq\left\|x_{n}-z\right\|^{2}-\left\|x_{n}-u_{n}\right\|^{2}+\lambda_{n} M\left\|\nabla f_{\alpha_{n}}\left(x_{n}\right)-\nabla f_{\alpha_{n}}(z)\right\| .
\end{aligned}
$$

By (3.17) and (3.19), we obtain

$$
\begin{aligned}
\left\|x_{n+1}-z\right\|^{2} & \leq \theta_{n}\left\|x_{n}-z\right\|^{2}+\left(1-\theta_{n}\right)\left\|u_{n}-z\right\|^{2}+\varepsilon_{n} M \\
& \leq \theta_{n}\left\|x_{n}-z\right\|^{2}+\left(1-\theta_{n}\right)\left[\left\|x_{n}-z\right\|^{2}-\left\|x_{n}-u_{n}\right\|^{2}\right.
\end{aligned}
$$




$$
\begin{aligned}
& \left.+\lambda_{n} M\left\|\nabla f_{\alpha_{n}}\left(x_{n}\right)-\nabla f_{\alpha_{n}}(z)\right\|\right]+\varepsilon_{n} M \\
= & \left\|x_{n}-z\right\|^{2}-\left(1-\theta_{n}\right)\left\|x_{n}-u_{n}\right\|^{2}+\left(\lambda_{n}\left\|\nabla f_{\alpha_{n}}\left(x_{n}\right)-\nabla f_{\alpha_{n}}(z)\right\|+\varepsilon_{n}\right) M .
\end{aligned}
$$

It follows that

$$
\left(1-\theta_{n}\right)\left\|x_{n}-u_{n}\right\|^{2} \leq\left\|x_{n+1}-x_{n}\right\|+\left(\lambda_{n}\left\|\nabla f_{\alpha_{n}}\left(x_{n}\right)-\nabla f_{\alpha_{n}}(z)\right\|+\varepsilon_{n}\right) M .
$$

Since $\liminf _{n \rightarrow \infty}\left(1-\theta_{n}\right)>0, \lim _{n \rightarrow \infty} \varepsilon_{n}=0, \lim _{n \rightarrow \infty}\left\|x_{n+1}-x_{n}\right\|=0,\left\{\nabla f\left(x_{n}\right)\right\}$ is bounded and $\lim _{n \rightarrow \infty} \lambda_{n}=0$, we derive that

$$
\lim _{n \rightarrow \infty}\left\|x_{n}-u_{n}\right\|=0
$$

Setting $y_{n}=T_{t} u_{n}$, from (3.16), we have

$$
\begin{aligned}
\left\|x_{n}-y_{n}\right\| \leq & \left\|x_{n+1}-x_{n}\right\|+\left\|x_{n+1}-y_{n}\right\| \\
\leq & \left\|x_{n+1}-x_{n}\right\|+\left\|\theta_{n} x_{n}+\left(1-\theta_{n}\right)\left[\varepsilon_{n} \gamma g\left(x_{n}\right)+\left(I-\varepsilon_{n} \mu B\right) y_{n}\right]-y_{n}\right\| \\
\leq & \left\|x_{n+1}-x_{n}\right\|+\theta_{n}\left\|x_{n}-y_{n}\right\|+\left(1-\theta_{n}\right)\left\|\varepsilon_{n} \gamma g\left(x_{n}\right)-\varepsilon_{n} \mu B T_{t} u_{n}\right\| \\
\leq & \left\|x_{n+1}-x_{n}\right\|+\theta_{n}\left\|x_{n}-y_{n}\right\|+\left(1-\theta_{n}\right)\left(\varepsilon_{n} \gamma \beta\left\|x_{n}-u_{n}\right\|\right. \\
& \left.+\varepsilon_{n}\left\|\gamma g\left(u_{n}\right)-\mu B T_{r} u_{n}\right\|\right) .
\end{aligned}
$$

Thus,

$$
\left\|x_{n}-y_{n}\right\| \leq \frac{1}{\left(1-\theta_{n}\right)}\left\|x_{n+1}-x_{n}\right\|+\varepsilon_{n} \gamma \beta\left\|x_{n}-u_{n}\right\|+\varepsilon_{n}\left\|\gamma g\left(u_{n}\right)-\mu B T_{r} u_{n}\right\| .
$$

From $\lim _{n \rightarrow \infty} \varepsilon_{n}=0$ and $\left\{u_{n}\right\}$ is bounded, we obtain

$$
\lim _{n \rightarrow \infty}\left\|x_{n}-y_{n}\right\|=0
$$

By (3.20) and (3.21), we also have

$$
\lim _{n \rightarrow \infty}\left\|y_{n}-u_{n}\right\|=\lim _{n \rightarrow \infty}\left(\left\|y_{n}-x_{n}\right\|+\left\|x_{n}-u_{n}\right\|\right)=0 .
$$

Next, we prove

$$
\limsup _{n \rightarrow \infty}\left\langle(\gamma g-\mu B) x^{*}, y_{n}-x^{*}\right\rangle \leq 0, \quad x^{*} \in P_{\Omega} g\left(x^{*}\right) .
$$

Indeed, we can choose a subsequence $\left\{u_{n_{i}}\right\}$ of $\left\{y_{n}\right\}$ such that

$$
\limsup _{n \rightarrow \infty}\left\langle(\gamma g-\mu B) x^{*}, y_{n}-x^{*}\right\rangle \leq \limsup _{n \rightarrow \infty}\left\langle(\gamma g-\mu B) x^{*}, y_{n_{i}}-x^{*}\right\rangle \leq 0 \text {. }
$$

Without loss of generality, we may further assume that $y_{n_{i}}-\tilde{x}$. By the same argument as that of Step 4 from Theorem 3.4, we can deduce that $\tilde{x} \in \Omega$. Therefore,

$$
\limsup _{n \rightarrow \infty}\left\langle(\gamma g-\mu B) x^{*}, y_{n}-x^{*}\right\rangle \leq\left\langle(\gamma g-\mu B) x^{*}, \tilde{x}-x^{*}\right\rangle \leq 0 .
$$


From (3.16), we have

$$
\begin{aligned}
\left\|x_{n+1}-x^{*}\right\|^{2} & \theta_{n}\left\|x_{n}-x^{*}\right\|^{2}+\left(1-\theta_{n}\right)\left\|y_{n}-x^{*}+\varepsilon_{n} \gamma g\left(x_{n}\right)-\varepsilon_{n} \mu B y_{n}\right\|^{2} \\
= & \theta_{n}\left\|x_{n}-x^{*}\right\|^{2}+\left(1-\theta_{n}\right)\left(\left\|y_{n}-x^{*}\right\|^{2}+2 \varepsilon_{n} \gamma\left\langle g\left(x_{n}\right), y_{n}-x^{*}\right\rangle\right. \\
& \left.-2 \varepsilon_{n}\left\langle\mu B y_{n}, y_{n}-x^{*}\right\rangle+\varepsilon_{n}^{2}\left\|\gamma g\left(x_{n}\right)-\mu B y_{n}\right\|^{2}\right) \\
= & \theta_{n}\left\|x_{n}-x^{*}\right\|^{2}+\left(1-\theta_{n}\right)\left(\left\|T_{r} u_{n}-x^{*}\right\|^{2}+2 \varepsilon_{n} \gamma\left\langle g\left(x_{n}\right)-g\left(x^{*}\right), y_{n}-x^{*}\right\rangle\right. \\
& -2 \varepsilon_{n}\left\langle\mu B y_{n}-\mu B x^{*}, y_{n}-x^{*}\right\rangle+2 \varepsilon_{n}\left\langle\gamma g\left(x^{*}\right)-\mu B x^{*}, y_{n}-x^{*}\right\rangle \\
& \left.+\varepsilon_{n}^{2}\left\|\gamma g\left(x_{n}\right)-\mu B y_{n}\right\|^{2}\right) \\
\leq & \theta_{n}\left\|x_{n}-x^{*}\right\|^{2}+\left(1-\theta_{n}\right)\left(\left\|u_{n}-x^{*}\right\|^{2}+2 \varepsilon_{n} \gamma\left\|g\left(x_{n}\right)-g\left(x^{*}\right)\right\|\left\|T_{r} u_{n}-x^{*}\right\|\right. \\
& -2 \varepsilon_{n}\left\|\mu B y_{n}-\mu B x^{*}\right\|\left\|T_{r} u_{n}-x^{*}\right\|+2 \varepsilon_{n}\left\langle\gamma g\left(x^{*}\right)-\mu B x^{*}, y_{n}-x^{*}\right\rangle \\
& \left.+\varepsilon_{n}^{2}\left\|\gamma g\left(x_{n}\right)-\mu B T_{r} u_{n}\right\|^{2}\right) \\
\leq & \theta_{n}\left\|x_{n}-x^{*}\right\|^{2}+\left(1-\theta_{n}\right)\left(\left\|x_{n}-x^{*}\right\|^{2}+2 \varepsilon_{n} \gamma \beta\left\|x_{n}-x^{*}\right\|\left\|u_{n}-x^{*}\right\|\right. \\
& \left.-2 \varepsilon_{n} \tau\left\|x_{n}-x^{*}\right\|^{2}+2 \varepsilon_{n}\left\langle\gamma g\left(x^{*}\right)-\mu B x^{*}, y_{n}-x^{*}\right\rangle+\varepsilon_{n}^{2}\left\|\gamma g\left(x_{n}\right)-\mu B T_{r} u_{n}\right\|^{2}\right) \\
\leq & \theta_{n}\left\|x_{n}-x^{*}\right\|^{2}+\left(1-\theta_{n}\right)\left(\left\|x_{n}-x^{*}\right\|^{2}+2 \varepsilon_{n} \gamma \beta\left\|x_{n}-x^{*}\right\|^{2}-2 \varepsilon_{n} \tau\left\|x_{n}-x^{*}\right\|^{2}\right. \\
& \left.+2 \varepsilon_{n}\left\langle\gamma g\left(x^{*}\right)-\mu B x^{*}, y_{n}-x^{*}\right\rangle+\varepsilon_{n}^{2} M\right) \\
= & \left(1-2 \varepsilon_{n}(\gamma \beta-\tau)\right)\left\|x_{n}-x^{*}\right\|^{2}+2\left(1-\theta_{n}\right) \varepsilon_{n}\left\langle\gamma g\left(x^{*}\right)-\mu B x^{*}, y_{n}-x^{*}\right\rangle \\
& +\varepsilon_{n}^{2}\left(1-\theta_{n}\right) M \\
= & \left.1-\sigma_{n}\right)\left\|x_{n}-x^{*}\right\|^{2}+\delta_{n} \sigma_{n}, \\
\leq & \\
&
\end{aligned}
$$

where $\sigma_{n}=2 \varepsilon_{n}(\gamma \beta-\tau)$ and $\delta_{n}=\frac{\left(1-\theta_{n}\right)}{2(\gamma \beta-\tau)}\left\langle\gamma g\left(x^{*}\right)-\mu B x^{*}, y_{n}-x^{*}\right\rangle+\frac{\varepsilon_{n}\left(1-\theta_{n}\right)}{\gamma \beta-\tau} M$. It is clear that $\sum_{n=0}^{\infty} \sigma_{n}=\infty$ and $\sup _{n \rightarrow \infty} \delta_{n} \leq 0$. Hence, all the conditions of Lemma 2.17 are satisfied. Therefore, we immediately deduce that $\lim _{n \rightarrow \infty} x_{n}=x^{*}$.

Remark 3.7 If we take $g=0$, by the similar argument as that in Theorem 3.6, we deduce immediately that $x^{*}$ is a particular solution of the variational inequality (3.1). This completes the proof.

\section{Application in the multiple-set split feasibility problem}

Recall that the multiple-set split feasibility problem (MSSFP) [4] is to find a point $x^{*}$ such that

$$
x^{*} \in C=\bigcap_{i=1}^{N} C_{i} \text { and } A x^{*} \in Q=\bigcap_{j=1}^{M} Q_{j} \text {, }
$$

where $N, M \geq 1$ are integers, $C_{i}$ and $Q_{j}$ are closed convex subsets of Hilbert spaces $H_{1}$ and $H_{2}$, and $A: H_{1} \rightarrow H_{2}$ is a bounded linear operator. The special case where $N=M=1$, called the split feasibility problem (1.1), was introduced by Censor and Elfving [4] for modeling phase retrieval and other image restoration problems. 
Let $\Gamma$ be the solution set of SFP, and let $\gamma>0$. Assume that $x^{*} \in \Gamma$. Thus, $A x^{\prime \prime} \in Q_{1}$ which implies the equation $\left(I-P_{Q_{1}}\right) A x^{*}=0$ which in turn implies the equation $\gamma A^{*}\left(I-P_{Q_{1}}\right) A x^{*}=$ 0 , and hence the fixed point equation $\left(I-\gamma A^{*}\left(I-P_{Q_{1}}\right) A\right) x^{*}=x^{*}$. Requiring that $x^{*} \in C_{1}$, we consider the fixed point equation

$$
P_{C_{1}}\left(I-\gamma A^{*}\left(I-P_{Q_{1}}\right) A\right) x^{*}=x^{*} .
$$

It is claimed that the solutions of the fixed point equation (4.2) are exactly the solution of the SFP. According the Byrne [2] and Xu [17], we obtain the following proposition.

Proposition 4.1 Given $x^{*} \in H_{1}, x^{*}$ solves the SFP if and only if $x^{*}$ solves the fixed point (4.2).

From this proposition, we can easily obtain that MSSFP (4.1) is equivalent to a common fixed point problem of finitely many nonexpansive mappings, as we show in the following.

Decompose MSSFP into $N$ subproblems $(1 \leq i \leq N)$ :

$$
x_{i}^{*} \in C_{i}, \quad A x_{i}^{* \prime} \in Q=\bigcap_{j=1}^{M} Q_{j} .
$$

Next, we define a mapping $T_{i}$ as follows:

$$
T_{i} x=P_{C_{i}}\left(I-\gamma_{i} \nabla g\right) x=P_{C_{i}}\left(I-\gamma_{i} \sum_{j=1}^{M} \beta_{j} A^{*}\left(I-P_{Q_{j}}\right) A\right) x,
$$

where the proximity function $g$ is defined by

$$
g(x)=\frac{1}{2} \sum_{j=1}^{M} \beta_{j}\left\|A x-P_{Q_{j}} A x\right\|^{2},
$$

where $\{\beta\}_{j=1}^{M}$ are such that $\beta_{j}>0$. Consider the minimization of $g$ over $C$ :

$$
\min _{x \in C} g(x)=\min _{x \in C} \frac{1}{2} \sum_{j=1}^{M} \beta_{j}\left\|A x-P_{Q_{j}} A x\right\|^{2} .
$$

Observe that the gradient $\nabla g$ is

$$
\nabla g(x)=\sum_{j=1}^{M} \beta_{j} A^{*}\left(I-P_{Q_{j}}\right) A x,
$$

which is $L$-Lipschitz continuous with the constant $L=\sum_{j=1}^{M} \beta_{j}\|A\|^{2}$ and thus $\nabla g(x)$ is $\frac{1}{L}$-ism. It is claimed that if $0<\gamma_{i} \leq 2 / L, T_{i}$ is nonexpansive. Therefore, fixed point algorithms for nonexpansive mappings can be applied to MSSFP (4.1).

From Algorithm 3.1, Algorithm 3.3 and Proposition 4.1, we consider our results on the optimization method for solving MSSFP (4.1), and obtain the following two algorithms. 
Algorithm 4.2 For an arbitrary initial point $x_{0}$, we define a sequence $\left\{x_{n}\right\}_{n \geq 0}$ iteratively

$$
x_{n}=(I-t \mu B) T_{r}\left[P_{C_{N}}(I-\gamma \nabla g)\right] \cdots\left[P_{C_{1}}(I-\gamma \nabla g)\right] x_{n}, \quad \forall t \in(0,1) \text {, }
$$

for all $n \geq 0, T_{r}$ is defined by Lemma 2.8 and $\nabla g$ is introduced in (4.3).

Algorithm 4.3 For an arbitrary initial point $x_{0}$, we define a sequence $\left\{x_{n}\right\}_{n \geq 0}$ iteratively

$$
x_{n}=\left\{\varepsilon_{n} \gamma f+\left(I-\varepsilon_{n} \mu B\right) T_{r}\left[P_{C_{N}}(I-\gamma \nabla g)\right] \cdots\left[P_{C_{1}}(I-\gamma \nabla g)\right]\right\} x_{n},
$$

for all $n \geq 0$, where $\left\{\varepsilon_{n}\right\}$ are two real sequences in $[0,1], T_{r}$ is defined by Lemma 2.8 and $\nabla g$ is introduced in (4.3)

In addition, we would like to point out that Algorithm 4.3 includes Algorithm 4.2 as a special case due to the fact that the contraction $f$ is a possible nonself-mapping. According to Theorem 3.4, we obtain the following theorem.

Theorem 4.4 Let $C$ be a nonempty closed convex subset of a real Hilbert space $H$. Let $B$ be a $k$-Lipschitz and $\eta$-strongly monotone operator on $H$ with $k>0, \eta>0$ and $0<\mu<2 \eta / k^{2}$, and the sequence of $\left\{\alpha_{n}\right\}$ and $\left\{\gamma_{n}\right\}$ satisfy the conditions (i)-(iv) in Lemma 2.14. Let $F_{1}, F_{2}$ : $C \times C \rightarrow R$ be two bifunctions which satisfy the conditions (f1)-(f4), (h1)-(h3) and (H) in Lemma 2.8. Let $f: C \rightarrow H$ be a $\beta$-contraction. Assume $\Omega:=\Gamma \cap \operatorname{MEP}\left(F_{1}, F_{2}\right) \neq \emptyset$, $\Gamma$ is the solution set of MSSFP (4.1). Then the sequence $\left\{x_{n}\right\}$ generated by implicit Algorithm 4.3 converges in norm, as $\varepsilon_{n} \rightarrow 0$, to the unique solution $x^{*}$ of the variational inequality (3.1). In particular, if we take $g=0$, then the sequence $\left\{x_{n}\right\}$ defined by Algorithm 4.3 converges in norm, as $\varepsilon_{n} \rightarrow 0$, to the unique solution $x^{*}$ of the variational inequality (3.1).

Proof Let

$$
U=T_{N} \cdots T_{1}=\left[P_{C_{N}}(I-\gamma \nabla g)\right] \cdots\left[P_{C_{1}}(I-\gamma \nabla g)\right]
$$

Then, as the composition of finitely many nonexpansive mappings, $U$ is nonexpansive. Also Algorithm 4.3 can be written as

$$
x_{n}=\left[\varepsilon_{n} \gamma g+\left(I-\varepsilon_{n} \mu B\right) T_{r} U\right] x_{n}, \quad x \in C .
$$

Since $T_{r}$ and $U$ are nonexpansive, and following the proof of Theorem 3.4, we obtain the sequence $\left\{x_{n}\right\}$ converges strongly to a fixed point of $U$ which is also a common fixed point of $T_{1}, \ldots, T_{N}$ or a solution of MSSFP (4.1).

From Theorem 3.6, we introduce an explicit algorithm for finding a common fixed point and for solving the variational inequality (3.1) and multiple set feasibility problem (4.1). This scheme is obtained by discretizing the implicit scheme (4.8).

Theorem 4.5 Let $C$ be a nonempty closed convex subset of a real Hilbert space H. Let B be a $k$-Lipschitz and $\eta$-strongly monotone operator on $H$ with $k>0, \eta>0$ and $0<\mu<2 \eta / k^{2}$, and the sequence of $\left\{\alpha_{n}\right\}$ and $\left\{\gamma_{n}\right\}$ satisfy the conditions (i)-(iv) in Lemma 2.14. Let $F_{1}, F_{2}$ : 
$C \times C \rightarrow R$ be two bifunctions which satisfy the conditions (f1)-(f4), (h1)-(h3) and (H) in Lemma 2.8. Let $f: C \rightarrow H$ be a $\beta$-contraction. Assume $\Omega:=\Gamma \cap \operatorname{MEP}\left(F_{1}, F_{2}\right) \neq \emptyset, \Gamma$ is the solution set of MSSFP (4.1). For given $\forall x_{0} \in C$, let the sequence $\left\{x_{n}\right\}$ generated by

$$
\begin{aligned}
x_{n+1}= & \theta_{n} x_{n}+\left(1-\theta_{n}\right)\left\{\varepsilon_{n} \gamma f+\left(I-\varepsilon_{n} \mu B\right)\right. \\
& \left.\times T_{r}\left[P_{C_{N}}(I-\gamma \nabla g)\right] \cdots\left[P_{C_{1}}(I-\gamma \nabla g)\right]\right\} x_{n}, \quad n \geq 0,
\end{aligned}
$$

where $\left\{\varepsilon_{n}\right\}$ and $\left\{\theta_{n}\right\}$ are two sequences in $[0,1]$, satisfy the following conditions:

(i) $\lim _{n \rightarrow \infty} \varepsilon_{n}=0$ and $\sum_{n=1}^{\infty} \varepsilon_{n}=\infty$;

(ii) $0<\liminf _{n \rightarrow \infty} \theta_{n} \leq \lim \sup _{n \rightarrow \infty} \theta_{n}<1$;

(iii) $\lim _{n \rightarrow \infty} \lambda_{n}=0$.

Then the sequence $\left\{x_{n}\right\}$ converges strongly to $x^{*}$ which is the unique solution of the variational inequality (3.1). In particular, if $f=0$, then the sequence $\left\{x_{n}\right\}$ generated by

$$
x_{n+1}=\theta_{n} x_{n}+\left(1-\theta_{n}\right)\left\{\left(I-\varepsilon_{n} \mu B\right) T_{r}\left[P_{C_{N}}(I-\gamma \nabla g)\right] \cdots\left[P_{C_{1}}(I-\gamma \nabla g)\right]\right\} x_{n}, \quad n \geq 0,
$$

converges strongly to a solution of the following variational inequality:

$$
\left\langle\mu B x^{*}, x-x^{*}\right| \geq 0, \quad \forall x \in \Gamma \text {. }
$$

Proof Following the assumption of (4.6), (4.8) can be written as

$$
x_{n+1}=\theta_{n} x_{n}+\left(1-\theta_{n}\right)\left\{\varepsilon_{n} \gamma f+\left(I-\varepsilon_{n} \mu B\right) T_{r} U\right\} x_{n}, \quad n \geq 0 .
$$

Since $T_{r}$ and $U$ are nonexpansive, following the proof of Theorem 3.6, we can easily claim that the sequence $\left\{x_{n}\right\}$ converges strongly to the common fixed point of $T_{r}$ which solves the mixed equilibrium problem $\left(\operatorname{MEP}\left(F_{1}, F_{2}\right)\right)$, and $U$ is a solution of MSSFP (4.1).

According to [22], we can obtain the following proposition.

Proposition 4.6 $x^{*}$ is a solution of MSSFP (4.1) if and only iff $\left(x^{*}\right)=0$.

Observe that if MSSFP(4.1) is consistent, then any solution $x$ is a minimizer of $f$ with minimum value zero. Note that a proximity function $f$ is as follows:

$$
f(x)=\frac{1}{2} \sum_{i=1}^{N} \alpha_{i}\left\|x-P_{C_{i}} x\right\|^{2}+\frac{1}{2} \sum_{j=1}^{M} \beta_{j}\left\|A x-P_{Q_{j}} A x\right\|^{2},
$$

where $\alpha_{i}>0$ for all $0<i \leq N$ and $\beta_{i}>0$ for all $0<i \leq M$. Then the gradient of $f$ is

$$
\nabla f(x)=\sum_{i=1}^{N} \alpha_{i}\left(I-P_{C_{i}}\right) x+\sum_{j=1}^{M} \beta_{j} A^{*}\left(I-P_{Q_{j}}\right) A x .
$$

It is claimed that the gradient $\nabla f$ is Lipschitz with the constant

$$
L^{\prime}=\sum_{i=1}^{N} \alpha_{i}+\sum_{j=1}^{M} \beta_{j}\|A\|^{2}
$$


To see this, we notice that projections and their complements are nonexpansive. Thus, both $I-P_{C_{i}}$ and $I-P_{Q_{j}}$ are nonexpansive for each $i$ and $j$. In addition, we can easily obtain that $\frac{1}{L^{\prime}} \nabla f$ is a nonexpansive mapping. Therefore, we can use the gradient projection method to solve the minimization problem:

$$
\min _{x \in \Omega} f(x)
$$

where $\Omega$ is a closed convex subset of $H_{1}$, whose intersection with the solution set of MSSFP is nonempty, and obtain a solution of the so-called constrained multiple set feasibility problem (CMSSFP):

$$
x \in \Omega \quad \text { such that } x^{*} \text { solves (4.1). }
$$

From Proposition 4.6 and Algorithm 3.3, we obtain the corresponding algorithm and the convergence theorems for MSSFP (4.1).

Algorithm 4.7 For an arbitrary initial point $x_{0}$, we define a sequence $\left\{x_{n}\right\}_{n \geq 0}$ iteratively

$$
x_{n+1}=\theta_{n} x_{n}+\left(1-\theta_{n}\right)\left\{\varepsilon_{n} \gamma g+\frac{1}{L^{\prime}}\left(I-\varepsilon_{n} \mu B\right) T_{r} \nabla f\right\} x_{n}
$$

for all $n \geq 0$, where $\left\{\varepsilon_{n}\right\}$ and $\left\{\theta_{n}\right\}$ are two real sequences in $[0,1], T_{r}$ is defined by Lemma 2.8 and $\nabla f$ is introduced in (4.9).

Theorem 4.8 Let $C$ be a nonempty closed convex subset of a real Hilbert space H. Let $B$ be a $k$-Lipschitz and $\eta$-strongly monotone operator on $H$ with $k>0, \eta>0$ and $0<\mu<2 \eta / k^{2}$, and the sequence of $\left\{\alpha_{n}\right\}$ and $\left\{\gamma_{n}\right\}$ satisfy the conditions (i)-(iv) in Lemma 2.14. Let $F_{1}, F_{2}$ : $C \times C \rightarrow R$ be two bifunctions which satisfy the conditions (f1)-(f4), (h1)-(h3) and (H) in Lemma 2.8. Let $g: C \rightarrow H$ be a $\beta$-contraction. Assume $\Omega:=\Gamma \cap \operatorname{MEP}\left(F_{1}, F_{2}\right) \neq \emptyset$, $\Gamma$ is the solution set of MSSFP (4.1). For given $\forall x_{0} \in C$, let the sequence $\left\{x_{n}\right\}$ generated by Algorithm 4.7 , where $\left\{\varepsilon_{n}\right\}$ and $\left\{\theta_{n}\right\}$ are two sequences in $[0,1]$, satisfy the following conditions:

(i) $\lim _{n \rightarrow \infty} \varepsilon_{n}=0$ and $\sum_{n=1}^{\infty} \varepsilon_{n}=\infty$;

(ii) $0<\liminf _{n \rightarrow \infty} \theta_{n} \leq \lim \sup _{n \rightarrow \infty} \theta_{n}<1$;

(iii) $\lim _{n \rightarrow \infty} \lambda_{n}=0$.

Then the sequence $\left\{x_{n}\right\}$ converges strongly to $x^{*}$ which is the unique solution of the variational inequality (3.1). In particular, if $g=0$, then the sequence $\left\{x_{n}\right\}$ generated by

$$
x_{n+1}=\theta_{n} x_{n}+\left(1-\theta_{n}\right)\left\{\frac{1}{L^{\prime}}\left(I-\varepsilon_{n} \mu B\right) T_{r} \nabla f\right\} x_{n}, \quad n \geq 0,
$$

converges strongly to a solution of the following variational inequality:

$$
\left\langle\mu B x^{*}, x-x^{*}\right\rangle \geq 0, \quad \forall x \in \Gamma .
$$

Proof From Proposition 4.6, we know that $\frac{1}{L^{\prime}} \nabla f$ is a nonexpansive mapping. Thus, using the proof of Theorem 3.4, we obtain that the sequence $\left\{x_{n}\right\}$ converges strongly to a fixed point of $\frac{1}{L^{\prime}} \nabla f$ or a solution of MSSFP (4.1), and this fixed point is a solution of the set $\operatorname{MEP}\left(F_{1}, F_{2}\right)$ of mixed equilibrium problem (1.3). 


\section{Competing interests}

The authors declare that they have no competing interests.

\section{Authors' contributions}

All authors contributed equally and significantly in writing this paper. All authors read and approved the final manuscript.

\section{Author details}

${ }^{1}$ School of Management, Tianjin University, Tianjin, 300072, China. ${ }^{2}$ College of Science, Civil Aviation University of China, Tianjin, 300300, China.

\section{Acknowledgements}

This work is supported in part by National Natural Science Foundation of China (71272148), the Ph.D. Programs Foundation of Ministry of Education of China (20120032110039) and China Postdoctoral Science Foundation (Grant No. 20100470783)

Received: 2 March 2012 Accepted: 27 November 2012 Published: 14 December 2012

\section{References}

1. Censor, Y, Elfving, T: A multiprojection algorithm using Bregman projections in a product space. Numer. Algorithms 8 , 221-239 (1994)

2. Byrne, C: Iterative oblique projection onto convex subsets and the split feasibility problem. Inverse Probl. 18, 441-453 (2002)

3. Byrne, C: A unified treatment of some iterative algorithms in signal processing and image reconstruction. Inverse Probl. 18, 103-120 (2004)

4. Censor, Y, Elfving, T, Kopf, N, Bortfeld, T: The multiple-sets split feasibility problem and its applications for inverse problems. Inverse Probl. 21, 2071-2084 (2005)

5. Lopez, G, Martin, V, Xu, HK: Iterative algorithms for the multiple-sets split feasibility problem. In: Censor, Y, Jiang, M, Wang, G (eds.) Biomedical Mathematics: Promising Directions in Imaging, Therapy Planning and Inverse Problems, pp. 243-279. Medical Physics Publishing, Madison (2009)

6. Qu, B, Xiu, N: A note on the CQ algorithm for the split feasibility problem. Inverse Probl. 21, 1655-1665 (2005)

7. Wang, F, Xu, HK: Cyclic algorithms for split feasibility problems in Hilbert spaces. Nonlinear Anal. (2011). doi:10.1016/j.na.2011.03.044

8. Xu, HK: A variable Krasnosel'skii-Mann algorithm and the multiple-set split feasibility problem. Inverse Probl. 22 2021-2034 (2006)

9. Jaiboona, C, Kumam, P: A general iterative method for addressing mixed equilibrium problems and optimization problems. Nonlinear Anal. 73, 1180-1202 (2010)

10. Kumam, $\mathrm{P}$, Jaiboon, C: Approximation of common solutions to system of mixed equilibrium problems, variational inequality problem, and strict pseudo-contractive mappings. Fixed Point Theory Appl. 2011, Article ID 347204 (2011)

11. Saewan, S, Kumam, P: A modified hybrid projection method for solving generalized mixed equilibrium problems and fixed point problems in Banach spaces. Comput. Math. Appl. 62, 1723-1735 (2011)

12. Saewan, S, Kumam, P: Convergence theorems for mixed equilibrium problems, variational inequality problem and uniformly quasi-asymptotically nonexpansive mappings. Appl. Math. Comput. 218, 3522-3538 (2011)

13. Xie, DP: Auxiliary principle and iterative algorithm for a new system of generalized mixed equilibrium problems in Banach spaces. Appl. Math. Comput. 218, 3507-3514 (2011)

14. Yao, YH, Noor, MA, Lioud, YC, Kang, SM: Some new algorithms for solving mixed equilibrium problems. Comput. Math. Appl. 60, 1351-1359 (2010)

15. Geobel, K, Kirk, WA: Topics in Metric Fixed Point Theory. Cambridge Studies in Advanced Mathematics, vol. 28 Cambridge University Press, Cambridge (1990)

16. Cianciaruso, F, Marino, G, Muglia, L, Hong, Y: A hybrid projection algorithm for finding solutions of mixed equilibrium problem and variational inequality problem. Fixed Point Theory Appl. 2010, Article ID 383740 (2010)

17. Xu, HK: Iterative methods for the split feasibility problem in infinite-dimensional Hilbert spaces. Inverse Probl. 26 Article ID 105018 (2010)

18. Nadezhkina, N, Takahashi, W: Weak convergence theorem by an extra gradient method for nonexpansive mappings and monotone mappings. J. Optim. Theory Appl. 128, 191-201 (2006)

19. Browder, FE: Fixed point theorems for noncompact mappings in Hilbert spaces. Proc. Natl. Acad. Sci. USA 43, 1272-1276 (1965)

20. Xu, HK: Iterative algorithms for nonlinear operators. J. Lond. Math. Soc. 2, 1-17 (2002)

21. Xu, HK: Viscosity approximation methods for nonexpansive mappings. J. Math. Anal. Appl. 298, $279-291$ (2004)

22. Yao, YH, Chen, RD, Marino, G, Liou, YC: Applications of fixed-point and optimization methods to the multiple-set split feasibility problem. J. Appl. Math. 2012, Article ID 927530 (2012) 